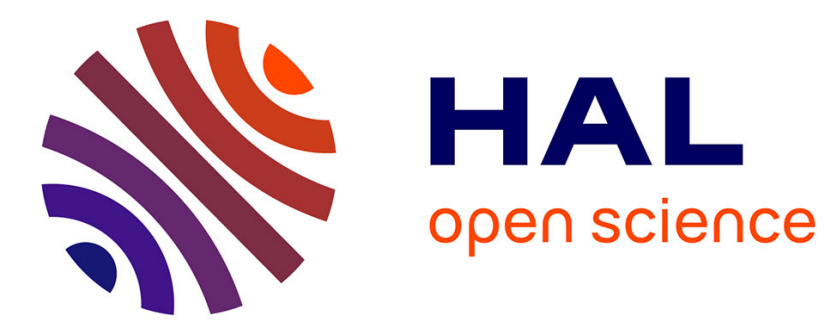

\title{
Politiques des mobilités à Angers : des conflits aux territoires
}

\author{
Jean-Pierre Wolff
}

\section{To cite this version:}

Jean-Pierre Wolff. Politiques des mobilités à Angers: des conflits aux territoires. Norois, 2000, 185

(1), pp.77 - 87. 10.3406/noroi.2000.7000 . hal-01814158

\section{HAL Id: hal-01814158 \\ https://hal-univ-tlse2.archives-ouvertes.fr/hal-01814158}

Submitted on 12 Jun 2018

HAL is a multi-disciplinary open access archive for the deposit and dissemination of scientific research documents, whether they are published or not. The documents may come from teaching and research institutions in France or abroad, or from public or private research centers.
L'archive ouverte pluridisciplinaire HAL, est destinée au dépôt et à la diffusion de documents scientifiques de niveau recherche, publiés ou non, émanant des établissements d'enseignement et de recherche français ou étrangers, des laboratoires publics ou privés. 


\section{Politiques des mobilités à Angers : des conflits aux territoires}

In: Norois. Nº185, 2000-1. Patrimoine et environnement. Les territoires du conflit. pp. 77-87.

\section{Résumé}

Les conflits environnementaux touchent, divisent et peuvent même créer de nouveaux territoires. Les infrastructures routières importantes sont à l'origine de conflits importants liés à la remise en cause du patrimoine, des paysages et des territoires. La réalisation prochaine d'une autoroute et le projet de rocade sud illustre cette problématique à Angers.

\section{Abstract}

Divisions, crises and the creation of new territories can sometimes originate from conflicts on environmental issues. The large infrastructure of roads is often at the origin of serious conflicts about the national heritage, landscape and territory. The impending construction of a highway and the project plan of a bypass south of Angers is an example that illustrates those

Citer ce document / Cite this document :

Wolff Jean-Pierre. Politiques des mobilités à Angers : des conflits aux territoires. In: Norois. N¹85, 2000-1. Patrimoine et environnement. Les territoires du conflit. pp. 77-87.

doi : $10.3406 /$ noroi.2000.7000

http://www.persee.fr/web/revues/home/prescript/article/noroi_0029-182X_2000_num_185_1_7000 


\title{
Politiques des mobilités à Angers : des conflits aux territoires
}

\author{
Jean-Pierre WOLFF \\ UMR 6590 CNRS ESO \\ Université d'Orléans \\ Domaine de la Source \\ Rue de Tours \\ 45072 Orléans Cédex 02
}

\section{Résumé}

Les conflits environnementaux touchent, divisent et peuvent même créer de nouveaux territoires. Les infrastructures routières importantes sont à l'origine de conflits importants liés à la remise en cause du patrimoine, des paysages et des territoires. La réalisation prochaine d'une autoroute et le projet de rocade sud illustre cette problématique à Angers.

\begin{abstract}
Divisions, crises and the creation of new territories can sometimes originate from conflicts on environmental issues. The large infrastructure of roads is often at the origin of serious conflicts about the national heritage, landscape and territory. The impending construction of a highway and the project plan of a bypass south of Angers is an example that illustrates those issues.
\end{abstract}

$\mathrm{E}$ - urbanisation rapide et extension périurbaine - furent et sont largement favorisées par les politiques de déplacement privilégiant le mode individuel (MERLIN 1991, DUPUY 1995). Parmi les conséquences multiples de ce type de transport, des effets négatifs se manifestent à travers le grignotage des espaces non urbanisés et son corollaire la détérioration des paysages. Si cette motorisation favorise pour certains des choix résidentiels, elle participe pour beaucoup d'autres, à la dégradation des conditions de vie liées à l'augmentation des pollutions atmosphérique, phonique et visuelle. La redynamisation des politiques de transport en commun, même si elle entend apporter une réponse globale aux questions de déplacement, passe souvent par l'implantation

\footnotetext{
Mots-clés : Environnement. Réscau. Transports. Territoires. Acteurs. Pouvoirs. Associations. Conflits. Key words : Invirunment. Tiansports. Nerworks. Territories. Actors. Powers. Associations. Conflicts.
} 
d'infrastructures lourdes induisant des effets secondaires non négligeables - empiétement au sol, intrusion paysagère plus ou moins importante -, certes moins dévoreurs que la multiplication des voiries routière et autoroutière, mais soulevant également des luttes importantes - LGV, extensions d'aéroport. Face à ces aménagements, émergent des processus de contestation pouvant déboucher sur des conflits plus ou moins importants et aux incidences territoriales non négligeables.

La cristallisation de ces revendications environnementales autour de projets et/ou de réalisations lourdes dans un espace donné, débouche-t-elle sur des processus de renforcement ou de négation de territoires, voire de territorialité ? Ce concept de territoire illustré par DI MEO (1996), se construit autour d'éléments différents l'espace, la société, l'idéologie, la culture, la politique, l'économie et l'environnement -, qui peuvent être activés par une menace lourde de conséquences comme la construction d'une centrale nucléaire, l'arrivée d'une autoroute dans une vallée étroite ou dans un quartier urbanisé, la création d'une ligne ferroviaire à grande vitesse ou l'agrandissement d'un port ou d'un aéroport. A travers quelques cas, il apparaît que les conflits liés aux politiques de déplacement refondent les territoires, en en dévitalisant certains, en en intégrant d'autres dans des ensembles dynamiques et en créant des territoires aux accessibilités différentes.

\section{La presse régionale révélatrice des conflits locaux}

Angers, agglomération moyenne à l'échelle européenne (Jeanneau 1974 et 1993), est un observatoire, parmi tant d'autres, des conflits environnementaux induits par les politiques d'aménagement. Ce travail s'appuie sur des enquêtes et la lecture systémique de la presse locale, d'Ouest France en l'occurrence, entre 1990 et 1997'. Les articles retenus se divisent en deux groupes essentiellement à travers les critères de conflictualité ou de consensualité. Tout aménagement est susceptible d'occasionner des dérangements, d'accroître la pression fiscale ou de concerner un large éventail de la population. Cependant la traduction de la mobilisation de groupes de personnes dépend d'une nette hiérarchisation des aménagements envisagés. Les articles consensuels sont deux à trois fois plus nombreux que les articles conflictuels selon l'année considérée et traitent avant tout de projets d'aménagement urbain, de restructuration d'un quartier ou d'une place et de création ou de modernisation d'équipements existant dans l'agglomération angevine. Certaines opérations auraient pu être à l'origine de conflits locaux notoires comme la réalisation du quartier Saint-Serge à proximité du centre d'Angers ou celle du quartier Front de Maine face au château. Mais il n'en a rien été. Les articles conflictuels concernent avant tout les projets de réalisation d'infrastructures routière et autoroutière et à l'inverse peu le projet de LGV évoqué au milieu des années 1990 ou celui de l'aéroport de Marcé.

1. Angers dispose de deux quotidiens régionaux. Le premier par son origine locale, Le Courrier de l'Ouest affiche des tendances conservatrices. Le second, Oucst France, premier quotidien régional en France en matière de diffusion et de penchant chrétien-social couvrant l'Oucst, est sans doute plus nuancé que le premier cité sur les réalités angevines er c’est pour cela qu'il a servi à notre travail. 
Ces conflits touchent et menacent des territoires urbains, périurbains et ruraux. A travers la presse locale et pour le cas angevin, les conditions débouchant sur des conflits sont les suivantes :

- les dérangements multiples occasionnés dans le cadre de vie ;

- le coût supposé supporté par les collectivités locales et donc par les habitants ;

- la sensibilisation à une question environnementale qui, sans toucher directement une partie de la population, laisse planer une menace plus générale de pollution et de dégradation du cadre de vie.

En ne retenant que l'année 1997, 263 articles ont été recensés qui peuvent se ventiler en quatre rubriques de taille différente par leur nombre. Il s'agit des :

- infrastructures routières et autoroutières (projets de contournement autoroutier, de construction d'une rocade sud, voirie) avec 101 articles;

- opérations urbanistiques (quartiers, lotissements, restructuration d'une place ou d'un secteur, patrimoine) avec 129 articles ;

- opérations d'aménagement liées au développement local avec 34 articles ;

- opérations d'aménagement support de l'environnement (espaces verts, rives, pollutions, air) avec 31 articles.

\section{Aménagements routiers lourds entre ville et périurbanisation}

Toujours pour cette année, la localisation des conflits se répartit comme pour les précédentes dans des secteurs géographiques qui sont à la charnière de l'urbain et du périurbain. Il s'agit de deux projets routiers - continuation autoroutière et rocade sud -, qui couperont les communes d'Avrillé, de Bouchemaine et de Saint-Gemmes-sur-Loire. Dans le détail, le projet autoroutier à l'ouest de l'agglomération d'Angers, devant permettre la continuation de l'A 11 entre Nantes et Paris, délesterait la voie express sur berge d'Angers. Long de 16,5 kilomètres, il s'inscrit dans des espaces bien différents qui vont de l'île Saint-Aubin, submersible trois mois par an, à Beaucouzé, commune périurbaine à la limite de la technopole, située à proximité du raccordement de l'autoroute en direction de Nantes, en passant par le quartier angevin des Capucins ensemble composite urbanistiquement et socialement, en pleine recomposition urbaine et fonctionnelle et par la ville-parc résidentielle d'Avrillé, (cf. Fig. $n^{\circ} 1$. Le secteur Capucins-Aérodrome dans l'agglomération angevine). Quant à la rocade sud dénommée parfois Grande Liaison Sud, elle doit décharger les boulevards urbains entre le pont de l'Atlantique sur la Maine, la Roseraie et la commune des Ponts de Cé, d'une partie de leur trafic de transit. Pour cela, cet axe nouveau prendrait naissance à l'échangeur autoroutier de l'A 11 au niveau de Beaucouzé, traverserait les quartiers résidentiels de Bouchemaine comme Pruniers, couperait la Maine et les prairies inondables classées en zone naturelle d'intérêt faunistique et floristique - ZNIEF —, poursuivrait en disloquant la commune de Saint-Gemmes-sur-Loire, pour rejoindre le contournement est de la ville d'Angers. 


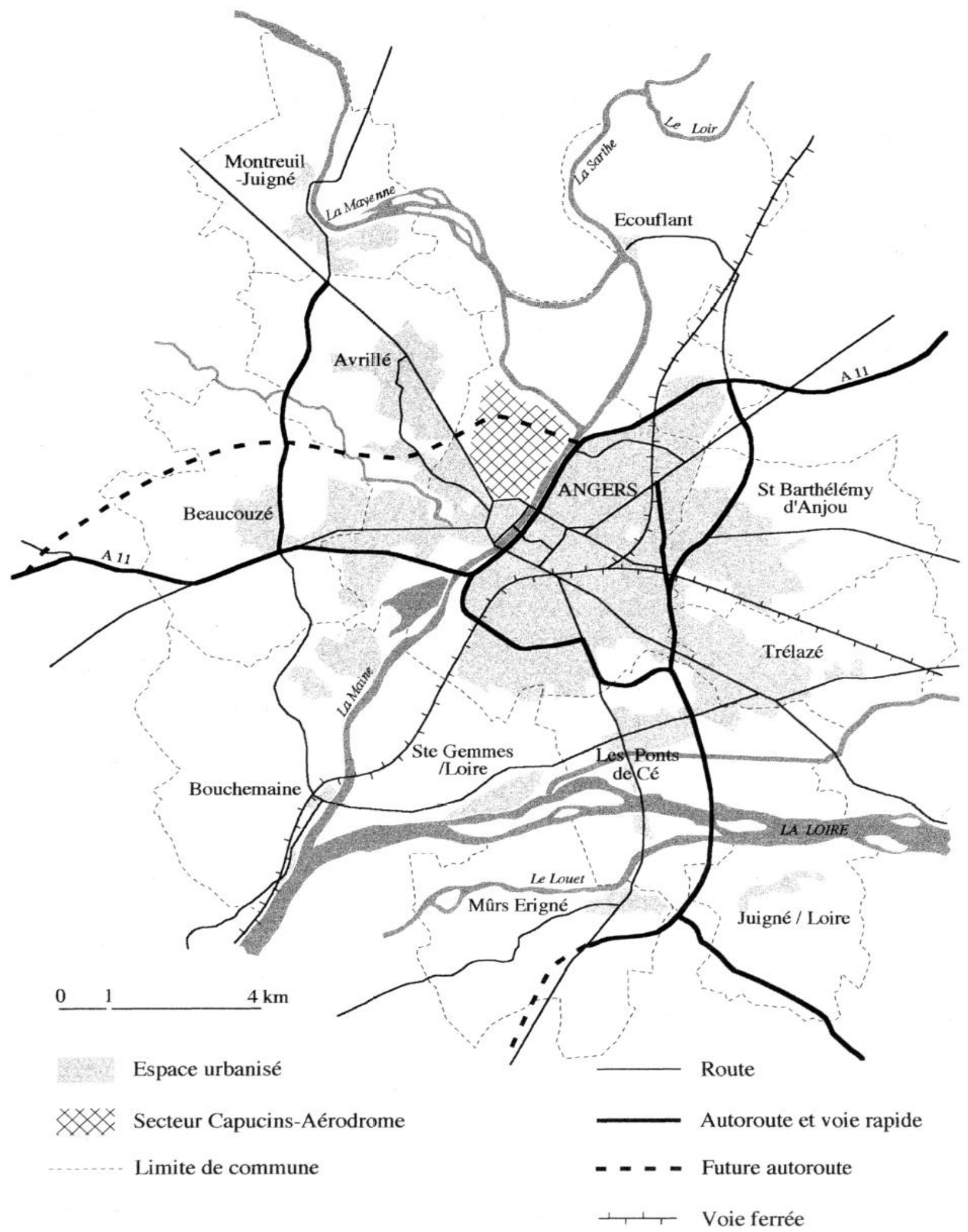

Figure 1 : Le secteur Capucins-Aérodrome dans l'agglomération angevine.

Les deux projets sont de nature différente, l'un est une continuation autoroutière prioritaire confiée à COFIROUTE et l'autre une voie express de contournement 
d'agglomération. Il est évident que ces infrastructures lourdes décomposent les territoires, mais en même temps elles les recomposent (Dupuy, 1995), en rapprochant des espaces éloignés, en créant des liens à travers le système automobile entrecroisant réseaux et territoires. Mais dans cette phase précédant celle de la réorganisation territoriale, la tension est vive face à ce changement potentiel important. Les rapports de force s'exacerbent (Barraque, 1993, Badie, 1995, Rey, 1997), en impliquant des acteurs publics et associatifs différents. Les territoires concernés sont caractérisés par la succession de deux phases d'expansion urbaine - périphérique puis périurbaine -, produisant une marqueterie paysagère et sociale diversifiée. Ces projets, dont les prémices remontent pour l'autoroute au début des années soixante et pour la rocade sud aux années 1975, ont connu de nombreuses variantes. Celles retenues visent un double objectif : soulager Angers d'une partie de cette circulation et favoriser l'accessibilité automobile de celle-ci et de son centre.

\section{A l'ouest, des mobilisations différentes contre l'autoroute A 11}

Le premier objet de conflictualité concerne la réalisation d'un maillon manquant pour achever l'A 11. Devant les menaces faites au cadre privilégié d'Avrillé, des associations de riverains se lancèrent dans la défense de l'environnement et du patrimoine immobilier. Dès 1975, se créé un Comité de défense contre le tracé de l'autoroute, à la base de la mobilisation de la population et de la municipalité d'Avrillé contre cette construction. En 1990, l'Association Contre le Tracé Autoroutier (ACTA) voit le jour et entreprend diverses démarches avec la municipalité (réunions publiques, rencontres avec les responsables du ministère de l'Equipement, balisage du tracé, plantation d'arbres...). La Coordination des $\Lambda$ ssociations Contre le Tracé Urbain Sud (CACTUS) dénonce ce projet qui non seulement détruirait le cadre de la ville-parc, mais également une partie du quartier Verneau sur la commune d'Angers. Les associations et la ville d'Avrillé se sont opposées à Angers, au district et à l'Etat, en invoquant en particulier la coupure induite par cette infrastructure autoroutière, source de nombreuses nuisances. En 1992, la municipalité organise un référendum local dont les résultats confirment amplement l'inquiétude de la population qui rejette massivement le contournement autoroutier. Le cadre politique national est à prendre en compte, c'est ainsi que le maire de droite et certaines associations s'en sont pris à l'Etat représenté par la DDE et non au gouvernement lorsque celui-ci était de même couleur politique, par contre dans le cas contraire, les revendications s'adressaient au pouvoir socialiste en place.

La population d'Avrillé est passée de 3000 à presque 13000 habitants entre 1960 et 1999, croissance liée au développement de lotissements pavillonnaires, ignorant ce projet en pointillé qui soulève la question de la pertinence territoriale pour ce secteur. L'autoroute ajouterait d'autres défauts à cette ville nouvelle, dont le développement décousu et distendu, sans grande cohérence d'ensemble autour d'un ancien noyau villageois, s'en trouverait accentué. Ceci remettrait en cause le principal souci des dernières municipalités qui a été de construire cette ville-parc autour d'éléments environnementaux et parrimoniaux comme l'étang Saint-Nicolas, l'aménagement du 
Bois-Assis, l'ouverture au public du parc de la Boissière, la création du parc GeorgesBrassens ou du golf public de Perrière. Cette ville manque d'unité, les quartiers composent des entités aux relations assez faibles et l'autoroute aggravera cela par une coupure supplémentaire, même si elle devra être recouverte sur un kilomètre.

L'absence de sentiment d'appartenance d'une grande partie de la population à la commune d'Avrillé, malgré la référence systématique au concept de ville-parc, soulignée par une enquête (Violier, 1996), est exacerbée par ce projet qui a de très fortes chances de se réaliser. L'intervention planificatrice de la ville d'Angers au niveau des terrains de l'ancien aérodrome accentuerait encore plus la fragmentation des territoires. Face à cette amputation d'une partie de la commune et avant une restructuration urbanistique et paysagère de l'ensemble, la mobilisation des associations et de la population débouche sur un sentiment récent d'appartenance à un secteur dont la concrétude s'affirme en négatif à travers cette menace. Le conflit, tout en soulignant l'éclatement du territoire communal (BADIE 1995), créé une communauté de défense qui, à terme, peut déboucher avec l'émergence d'une politique urbaine, sur une recomposition territoriale basée sur les échelons complémentaires et s'emboîtant du quartier à l'agglomération.

\section{Au sud, préoccupations écologiques et sociales contre la rocade}

Le second projet angevin, la rocade sud inscrite en 1976 dans le SDAU et confirmé dans le schéma directeur de la région angevine en 1996, touche plusieurs communes du sud de l'agglomération. Sur les sept scénarii présentés, a été retenu celui défini initialement en 1976 par le Syndicat Mixte de la Région Angevine (SMRA)². Ce tracé présente l'avantage de délester les quartiers sud d'Angers d'une partie de leur trafic par la construction d'une rocade peu éloignée de la ville mais coupant plusieurs communes de l'agglomération. Les problèmes soulevés sont de plusieurs ordres : environnementaux avec le franchissement de la vallée de la Maine, 270 hectares classés en ZNIEF, paysagers avec l'éventration des coteaux de Pruniers, économiques avec le démembrement de plusieurs unités horticoles de la commune de Saint-Gemmes-sur-Loire et l'atteinte portée au patrimoine immobilier. Ces conséquences nourrissent une situation conflictuelle aux nombreux acteurs - les communes touchées, la ville d'Angers, le district, plusieurs associations opposées et comités de défense du patrimoine et de l'environnement.

Les communes et les associations dénoncent ce projet. Les municipalités de Bouchemaine et de Saint-Gemmes-sur-Loire contestent la décision prise par le SMRA qui, le 1 er juillet 1996 par 58 voix pour, 20 contre, 2 abstentions et 3 bulletins nuls, adopte le Schéma directeur et donc avalise la rocade sud. Le conflit sera juridique et politique car est visé à travers le SMRA, le président du district et l'ancien maire d'Angers Jean Monnier - 1977/1999. Le désaccord se radicalise surtout dans deux communes pour des raisons différentes, comme la protection du patrimoine immobilier

2. AURA et SMRA, 1998, Schéma directeur de la région angevine, approuvé le 1.7.1996 et modifié le 12.3.1997. 
et foncier à Bouchemaine et la défense de l'outil de travail des horticulteurs et serriculteurs à Saint-Gemmes-sur-Loire. Bouchemaine avec plus de 6000 résidants, a connu une croissance démographique très rapide autour de trois noyaux anciens principaux dont l'un, Pruniers, est directement concerné par cette infrastructure routière. La composition sociale locale favorise l'adoption de deux stratégies qui se rejoignent dans le rejer du projet. L'une, assez classique par les moyens de mobilisation locale, recréée autour de Pruniers un sentiment très fort d'identité et d'appartenance à un même territoire. L'autre, par l'insertion sociale des résidents, trouve directement un écho dans les rouages de la notabilisation locale et a recours au système juridique pour faire aboutir son point de vue.

Lors des élections municipales de 1995, caisse de résonance des tensions locales, la liste conduite par un ancien membre de Génération écologie a remporté ses échéances locales marquées par les menaces pesant sur l'environnement et ceci malgré la présence de plusieurs listes de droite et de gauche. Lengagement, depuis cette date, des maires de Bouchemaine et de Saint-Gemmes-sur-Loire déplace le conflit sur la scène districale et trouve un écho auprès de certains élus de l'agglomération. Parallèlement à leur intervention, des associations agissent pour défendre les intérêts des habitants de ce secteur. La plus ancienne est l'Association pour la Protection de la Vallée de la Maine APROVAM -, créée en 1972 pour lutter contre la pollution de la vallée de la Maine. L'APROVAM intervient dans ce secteur de l'agglomération pour améliorer des situations critiques. Elle n'est pas liée à un territoire communal ou infra-communal. Par contre, dans ce cas, elle fédère sous le titre de Coordination des associations opposées au projet de rocade sud, plusieurs associations enracinées localement comme l'association du Val de Maine, les Amis du pont de Pruniers, l'Association des Hauts de Bouchemaine, l'Association le Vallon, situées à Bouchemaine, l'Association du camp de César, les Amis de Port-Thibault, localisćes à Saint-Gcmmcs-sur-Loirc ct enfin l'association Gaubourgs-Champs Saint-Martin d'Angers. Il faut souligner que l'Association de défense des habitants et des passants du boulevard sud, créée en 1995 à Angers et réclamant l'interdiction de la circulation des plus de 3,5 tonnes a été entendue par la Ville d'Angers qui a pris un décret favorable à cette requête en 1999, accentuant son souhait de voir se réaliser au plus vite cette rocade sud $^{3}$.

A côté de l'APROVAM, l'Association des Opposants au Tracé Val de Maine-Prunier AOT - née en 1996 après l'approbation de la rocade sud, n'appartient pas à cette coordination. L'AOT dénonce la passivité des autres associations et de la municipalité de Bouchemaine opposées à tous les tracés et qui refusent donc d'en choisir un. L'AOT considère que ce non-choix revient à en admettre tacitement un, qui, de plus léserait particulièrement les habitants de Pruniers. Cette association fortement implantée territorialement défend son environnement et soutient, pour cela, la réalisation d'une variante de cette rocade (tracé $n^{\circ} 2$ ), qui la gênerait peu, (cf. Fig. $n^{\circ} 2$. Les scénarii de la "Grande liaison Sud"). L'étude du tracé $n^{\circ} 3$, malgré la construction d'une tranchée recouverte et la remise en l'état du paysage, qui n'est pas garantie, indique que 219 habitations se trouvant à moins de 300 mètres de cette infrastructure, seraient touchées

3. Cf. Les boulevards sud sont interdits aux camions, Vivre à Angers, $n^{\circ} 227,1999$. 
et subiraient au moins une baisse de $30 \%$ de leur valeur immobilière. L'AOT prône donc la réalisation du tracé $n^{\circ} 2$, en arguant qu'à sa proximité, il n'y a que 14 maisons.

La défense des intérêts locaux soulève des antagonismes territoriaux illustrés par les conflits qui opposent la municipalité et plusieurs associations de Bouchemaine à l'AOT. Les relations entre cette association, la municipalité et l'APROVAM s'avèrent parfois tendues dans la mesure où celle-ci brise le système de défense de la commune. Dans ce cadre-là, la présence de trois noyaux anciens - Pruniers, Bouchemaine et la Pointe persiste en terme de territorialité, malgré ou à cause du développement de l'urbanisation dans les espaces interstitiels. Les conflits en cours, en dépit de l'arrêté du tribunal de Nantes, exacerbent les oppositions entre micro-territorialités, sous l'effet du NIMBY ${ }^{4}$. A l'intérieur de ces espaces contrôlés par une association se développe un esprit de résistance et de clocher qui accentue la division territoriale de la commune, donc d'une entité supérieure et englobante pourvue d'une représentativité institutionnelle. Il s'agit là, comme le note REY 1997 "d'une appropriation symbolique des lieux et d'immobilité, dans un plan ayant pour référence l'appartenance au sol et dans une vision américaine de la citoyenneté " $"$. La défense de l'environnement, des paysages et des territoires correspond à l'addition d'intérêts économiques, portée par une association soudée face à la perspective d'une dévalorisation patrimoniale.

\section{De l'environnement à la revendication territoriale}

Au-delà de ces deux cas, la question de notre problématique connaît des développements qui sont la somme d'enjeux, d'antagonismes et de compromis, souvent conflictuels et évoluant dans le temps. C'est ainsi qu'une des plus importantes luttes actuelles, se déroule dans la vallée pyrénéenne d'Aspe depuis une vingtaine d'années. L'abandon de la relation ferroviaire du Somport reliant Pau à Zaragoza, accéléra la revendication du projet de liaison routière rapide qui s'appuyait sur deux éléments, l'un en voie d'achèvement le tunnel routier entre Canfranc (province d'Aragon) et Les Forges d'Abel (Pyrénées-Atlantiques), long de huit kilomètres, l'autre, la modernisation de la voie d'accès, la R.N. 134, bien avancée mais en passe d'être remise en question.

La trilogie - acteurs/conflit/territoire - illustre d'une façon emblématique la question environnementale et territoriale. Pendant plus d'une décennie, deux camps se sont violemment opposés. Dans le premier, les défenseurs du milieu écologique, du tourisme doux, de l'économie pastorale et d'un développement local endogène, dans le second les tenants de la modernisation des infrastructures d'échange entre l'Occitanie et l'Aragon,

4. NIMBY - Not in my Back Yard - que l'on peut traduire "pas che\% moi ", exacerbe les mobilisations des individus ou des groupes qui se sentent menacer par des projets aussi bien importants que mineurs. Cette réaction de défense légitime dans des cas évidents de remise en cause d'équilibres écologiques ou paysagers par la réalisation de grosses opérations d'aménagement, apparaît disproportionnéc dans d'autres situations. Cette remise en cause aussi minime soit elle, s'apparente à une aggression inadmissible.

5. RrY (M.), 1997. - Conflits et représentations de la ville lors d'opérations d'urbanisme importantes à Toulouse, in Ville et environnement, Représentation et stratégies, Recherches urbaines $n^{\circ} 12$, CESURB, Talence, Bordeaux. 


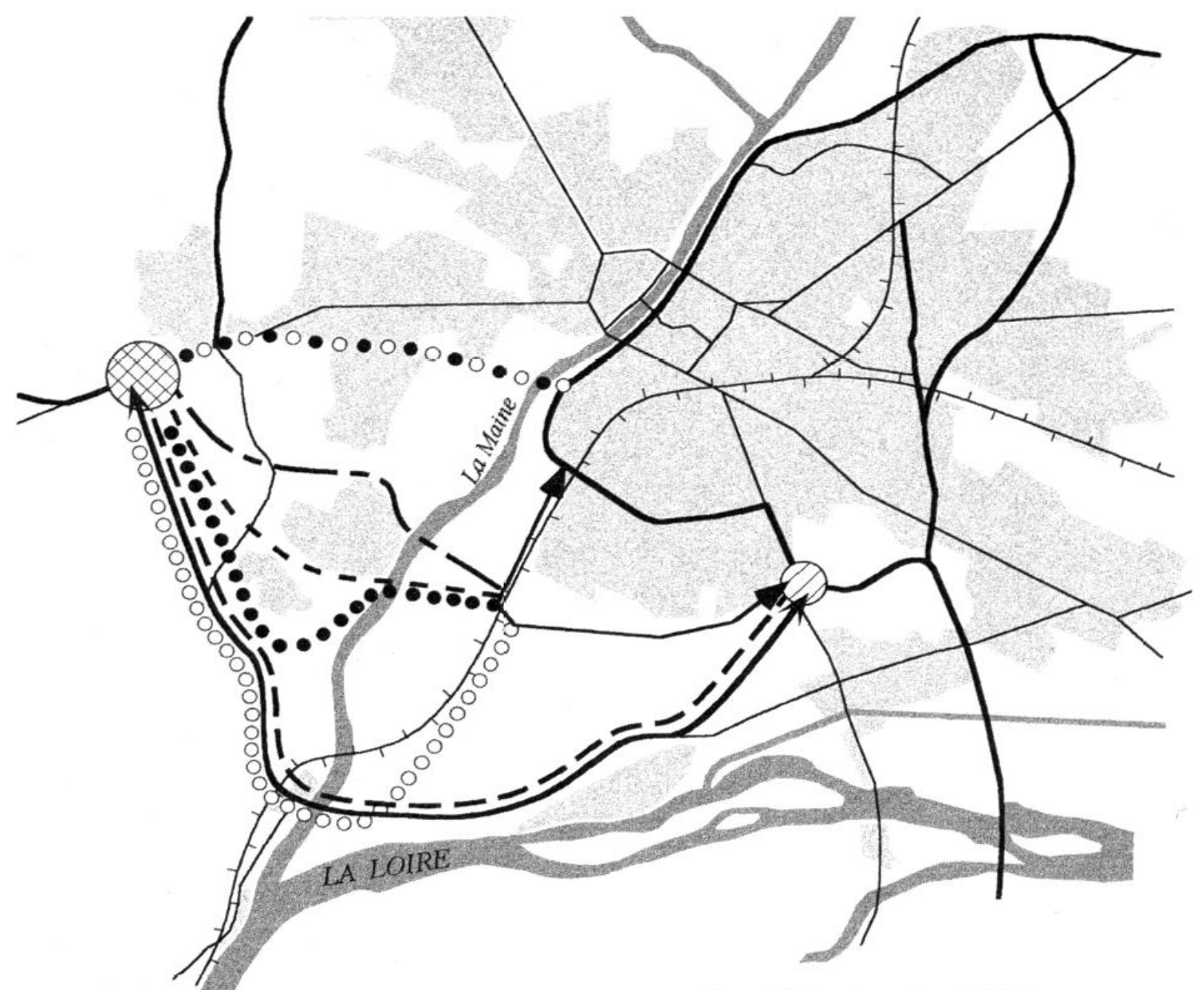

Les différents scénarii (S)

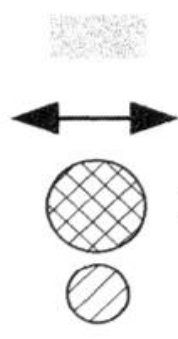

Espace urbain

Petite liaison sud

Echangeur de la Croix de Lorraine

Carrefour Mignon

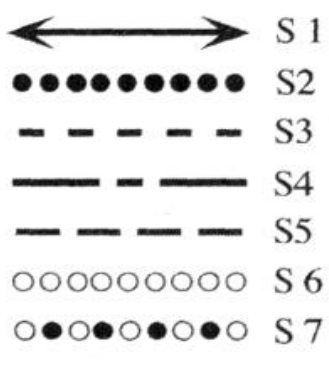

$2 \mathrm{~km}$

Figure 2 : Les scénarii de la " Grande Liaison Sud ».

entre le nord et le sud de l'Europe, censée apporter le progrès et l'essor économique à cette vallée enclavée. Actuellement face à l'acuité des problèmes de pollution et de sécurité dans des vallées encaissées et dans les tunnels, les fervents défenseurs de la route et des échanges routiers rejoignent dans les discours, les irréductibles "indiens ", après de multiples escarmouches de part et d'autres - appuis de milieux politiques, d'associations, d'acteurs économiques et scientifiques, manifestations pacifiques et violentes, obstructions et destructions, recours à la justice, emprisonnement à répétition du principal contestataire. Evoquer la réhabilitation de la ligne ferroviaire et le 
développement du ferroutage donnent raison aux associations qui se sont battues sur tous les plans et dont le conflit avait fait ressortir deux visions d'un territoire potentiellement profondément défiguré par ce ruban routier et sa déferlante de véhicules lourds.

Malgré les profondes divisions qui ont marqué cet espace-laboratoire et les risques d'implosion du système territorialisé, l'évolution des mentalités permet de rapprocher des mondes qui s'étaient affrontés pendant une quinzaine d'années. Les dangers de cette infrastructure routière à moitié achevée en 1999, rapprochent les positions des différents acteurs qui veulent préserver ce territoire des risques encourus, si le projet était mené à son terme. L'enjeu est certes différent des deux cas présentés précédemment, par son inscription géographique, sa finalité économique et ses retombés environnementales, mais il illustre la problématique de départ.

Dans les deux cas angevins, l'objet du conflit est un projet d'infrastructure routière lourde et qui ligue contre lui l'ensemble des populations susceptibles de voir leur environnement et leur patrimoine remis en cause. Cependant la contestation est différente en fonction des enjeux économiques et des intérêts de classe en présence. C'est ainsi qu'à Avrillé et à Bouchemaine, la population est nettement plus mobilisée qu'à la cité Verneau écornée par le futur contournement autoroutier. Les associations défendent leur cadre de vie en employant des moyens respectant la légalité, en veillant à ne pas troubler l'ordre public et en se constituant partie civile. Le recours contentieux s'érige en toile de fond des rapports sociaux et les associations recourent de plus en plus au droit qui apparaît comme l'alternative la plus crédible pour défendre une position environnementale et qui repose sur une légitimité de l'Etat face à la mise en place d'une certaine autonomie communale. Les phases de la monté en puissance du conflit se sont appuyées sur une période de militantisme, puis de politisation locale et enfin d'une action judiciaire.

Les territoires ou plutôt les champs du conflit ne sont pas géographiques stricto sensu, ils s'inscrivent avant tout dans le social. Ils participent aux questionnements de la géographie sociale. Ils dépendent également des rapports de force entre l'Etat, les municipalités, le district, les représentants économiques et les associations. En fonction des variantes présentées, discutées et retenues, les territoires se modifient sous l'effet de l'implication des populations les plus concernées et réactives à la situation en passe d'être entérinée. Le temps est un acteur non négligeable, la pression sur l'espace devenant plus forte à certains moments qu'à d'autres.

Ces territoires en devenir que sont par excellence les zones périphériques ou périurbaines sont soumis à des pressions économiques, techniques et idéologiques puissantes. Le conflit peut favoriser une territorialité basée sur la défense d'un environnement et d'un patrimoine commun. Mais en reprenant les deux exemples étudiés, les intervenants se mobilisent sur leur pré-carré. En cas de résultats positifs, ce sentiment de communauté gagnante peut se maintenir par la création d'un lieu symbolique, d'une mémoire collective, d'une fête..., par contre en cas d'échec la démobilisation guette et le ou les territoires s'effacent. 


\section{Références bibliographiques}

AURA et SMRA, 1998. - Schéma directeur de la région angevine, approuvé le 1.7 .1996 et modifié le 12.3.1997.

BADIE (B.), 1995. - La fin des territoires, Fayard, Paris.

BARraque (B.), 1993. - Le gouvernement local et l'environnement, in BIAREZ (S.) et NeVERs (JY.), (sous la direction de), Gouvernement local et politiques urbaines, CERAT, Grenoble.

Bieber (A.), Massot (M-H.) et Orfeuil (J-M.) 1993. - Prospective de la mobilité quotidienne, in BONNAFOUS A., PLASSARD F. ET VULIN B., (sous la direction de) Circuler demain, Paris, DATAR/L'Aube, pp. 157-184.

Di Mro (G.), 1996. - Les territoires du quotidien, L'Harmattan, Géographie sociale, Paris.

Di MEo (G.), 1996. - Géographie sociale et territoires, Nathan, Paris.

DupuY (G.), 1995. - Les territoires de l'automobile, Anthropos, Paris.

JeANNEAU (J.), 1974. - Angers et son agglomération, Les villes françaises, in Notes et études de la documentation française, Paris.

Jeanneau (J.), 1993. - Les villes de l'Anjou, Angers, Cholet, Saumur au milieu du XXe siècle, Ouest éditions, Nantes.

IFEN, 1998. - L'environnement en France, La Découverte, Orléans.

LeFeVRe (C.) et OFFner (J-M.), 1991. - Les transports urbains en question, CELSE, Paris.

MERLIN (P.), 1991. - Géographie, économie et planification des transports, PUF, Paris, 472 p.

Orfeuil. (J-P.), 1996. - La mobilité urbaine, son coût, ses modalités de financement in Pumain (D.) et Godard (F.), (sous la direction de), Données urbaines, Anthropos, Paris, pp. 187-197.

REY (M.), 1997. - Conflits et représentations de la ville lors d'opérations d'urbanisme importantes à Toulouse, in Ville et environnement, Représentation et stratégies, Recherches urbaines $n^{\circ} 12$, CESURB, Talence, Bordeaux.

Violier (Ph.), 1996. - Identirés et territoires des communes de banlieue. L'excmple de l'agglomération d'Angers, Travaux et documents de l'IFRESI, Lille, pp. 223-234. 